\title{
BMJ Open Excess costs of type 2 diabetes and their sociodemographic and clinical determinants: a cross-sectional study using data from the German Health Interview and Examination Survey for Adults (DEGS1)
}

\author{
Hannah König (D) , ${ }^{1}$ A Rommel, ${ }^{2}$ Jens Baumert (D) , ${ }^{2}$ Christian Schmidt (D) , \\ Hans-Helmut König, ${ }^{1}$ Christian Brettschneider (D) , ${ }^{1}$ Alexander Konnopka ${ }^{1}$
}

To cite: König H, Rommel A, Baumert J, et al. Excess costs of type 2 diabetes and their sociodemographic and clinical determinants: a crosssectional study using data from the German Health Interview and Examination Survey for Adults (DEGS1). BMJ Open 2021;11:e043944. doi:10.1136/ bmjopen-2020-043944

- Prepublication history and additional supplemental material for this paper are available online. To view these files, please visit the journal online (http://dx.doi.org/10.1136/ bmjopen-2020-043944)

$\mathrm{CB}$ and $\mathrm{AK}$ are last authors.

Received 18 August 2020

Revised 22 January 2021 Accepted 06 April 2021

Check for updates

(c) Author(s) (or their employer(s)) 2021. Re-use permitted under CC BY-NC. No commercial re-use. See rights and permissions. Published by BMJ.

For numbered affiliations see end of article.

Correspondence to

Hannah König;

ha.koenig@uke.de

\section{ABSTRACT}

Objectives The objectives of this study were to estimate the direct and indirect excess costs of type 2 diabetes mellitus (T2D) using data representative for the German adult population and to investigate the association of sociodemographic and clinical determinants with these excess costs.

Setting We calculated mean annual costs for individuals with T2D and a control group without diabetes, using data on healthcare utilisation and productivity losses from the cross-sectional German Health Interview and Examination Survey for Adults. We adjusted for group differences using entropy balancing and estimated excess costs for total, direct, indirect costs and additional cost categories using generalised linear models. We performed subgroup analyses to investigate the association of sociodemographic (age, sex and education) and clinical determinants (diabetes duration, glycaemic index and complications) with excess costs.

Participants The final study sample included $n=325$ individuals with T2D and $n=4490$ individuals without diabetes in the age between 18 and 79 years.

Results Total excess costs amounted to $€ 927$, of which $€ 719$ were attributable to direct and $€ 209$ to indirect excess costs. Total costs were significantly increased by $28 \%$ for T2D compared with controls. Group differences in direct, outpatient and medication costs were statistically significant. Medication costs were $88 \%$ higher for T2D and had the highest share in direct excess costs. With respect to specific determinants, direct excess costs ranged from $€ 203$ for $4-10$ years diabetes duration to $€ 1405$ for diabetes complications. Indirect excess costs ranged from $€-544$ for $>10$ years diabetes duration to $€ 995$ for high education.

Conclusions T2D was associated with high costs, mainly due to direct costs. As pointed out by our results, diabetes complications and comorbidities have a large impact on the costs, leaving medication costs as main contributor of T2D excess costs.
Strengths and limitations of this study

- This study used data representative for the German population aged 18-79 to estimate direct and indirect excess costs of type 2 diabetes mellitus (T2D).

- The groups were adjusted for multiple sociodemographic and clinical covariates using entropy balancing.

- Although the data were comprehensive and of high quality, not all cost categories that would have been relevant for the costs of T2D could be assessed.

- The sample sizes in the subgroups were partly small due to the complete case analysis.

- We tested the robustness of our results through various sensitivity analyses with respect to outliers, model adjustments and age of the population.

\section{INTRODUCTION}

Diabetes mellitus is one of the main causes of disability and premature death worldwide. ${ }^{1}$ With an increasing trend over the past 30 years, diabetes mellitus was the fourth most common cause of the global disease burden in $2017 .^{2}$ The global prevalence of diabetes in adults was estimated to be $8.5 \%$ (7.9\% in women and $9.0 \%$ in men) in $2014 .{ }^{13}$ In the German general population, the prevalence of diagnosed and undiagnosed diabetes was $9.2 \%(8.6 \%$ in women and $9.9 \%$ in men), assessed in 2008-2011. ${ }^{4}$ With regards to type 2 diabetes mellitus (T2D), the prevalence of diagnosed T2D was $7.2 \%$ in Germany. ${ }^{5}$ Stratified by gender, the prevalence was almost equal between women (7.4\%) and men $(7.0 \%) .^{5}$ Prevalence estimates based on documented data were consistently higher than those from surveys. ${ }^{6-8}$ Overall, the number of individuals with T2D keeps growing in 
Germany, with an expected increase of at least $54 \%$ between 2015 and 2040. ${ }^{9}$

Worldwide, T2D is associated with increased healthcare costs. ${ }^{10} 11$ Thereby, the share of direct costs (ie, the costs of healthcare utilisation) in total costs is higher than the share of indirect costs (ie, the costs of reduced/lost productivity as a result of the disease).$^{1011}$ The majority of previous cost-of-illness (COI) studies have not reported excess costs (ie, the difference in mean costs between a group of individuals with diabetes and a control group of individuals without diabetes). ${ }^{10}$ The advantage of reporting excess costs is that the share of costs actually attributable to diabetes can be estimated more accurately.

As for Germany, the Robert Koch Institute (RKI) has established a diabetes surveillance, which will inform the public timely and continuously about the developments concerning diabetes using certain indicators, including direct costs (diabsurv.rki.de). ${ }^{12}$ In 2015, approximately $€ 7$.4 billion were attributable to the primary diagnosis of diabetes, representing $2.2 \%$ of all disease-related costs. ${ }^{12} 13$ With respect to studies reporting excess costs, the annual average direct excess costs of diabetes ranged from $€ 985$ to $€ 3256$ per person (all costs were inflated to 2011). ${ }^{14-20}$ For the direct excess costs of diabetes, the influence of sociodemographic ${ }^{141719}$ or clinical $^{17-20}$ determinants on excess costs was also investigated. Fewer studies reported indirect excess costs, ranging from $€ 1551$ to $€ 2122$ per person. ${ }^{1720}$ The majority of the studies estimated excess costs using claims data from statutory health insurance funds, ${ }^{14-19}$ while one study used survey data to estimate excess costs. ${ }^{20}$ Thereof, several studies were conducted using the same data source. ${ }^{17-19}$ Although the studies followed a population-based approach, they mostly focused on specific regional areas, ${ }^{15}{ }^{17-20}$ like Southern Germany, ${ }^{20}$ or several cities in Northwest Germany. ${ }^{15}$ Nevertheless, two studies conducted nationwide analyses, ${ }^{1416}$ with one study using claims data from a random sample of the German statutory health insurance ${ }^{14}$ and the other using claims data of all insured of the largest German sickness fund (Allgemeine Ortskrankenkasse, AOK) ${ }^{16}$

Although the excess costs of T2D have been previously investigated in Germany, the body of research for the population as a whole is scarce. In particular, to our knowledge, no indirect excess costs of T2D representative of the German population have been estimated yet. Furthermore, only one study used survey data to estimate the excess costs of T2D, while all other findings were based on claims data. Survey data have the advantage that they contain comprehensive information on the study sample, which includes sociodemographic characteristics, healthcare utilisation and comorbidities. So far, previous studies from Germany have only investigated the association of sociodemographic or clinical determinants with direct excess costs. Subsequently, diabetes will refer to T2D, unless otherwise stated.

This study adds to the research gap by using survey data that are representative of the general population aged
18-79 years in Germany. The aim of this study was to estimate the direct and indirect excess costs of T2D in adults in Germany with a specific focus on correlates with sociodemographic and clinical determinants of diabetes. First, we estimated direct and indirect excess costs of T2D for the German general population. Second, we analysed the excess costs of T2D by sociodemographic (age, gender and education level) and clinical (diabetes duration, glycaemic index, complication) subgroups.

\section{METHODS}

\section{Study sample}

We used cross-sectional data from the German Health Interview and Examination Survey for Adults (DEGS1), which was conducted from 2008 to 2011 by the RKI. ${ }^{21}$ The DEGS1 collected comprehensive health data, including information on health service utilisation and the results of medical examinations and laboratory tests. The study design and methods have been published previously. ${ }^{22-24}$

In DEGS1, the total sample was comprised of 8151 study participants in the age between 18 and 91 years. ${ }^{23}$ We included those participants who were aged between 18 and 79 years and completed the interview and examination part $(\mathrm{n}=7115)$ and excluded missing values related to (1) diabetes status $(n=36),(2)$ costs $(n=1218)$, (3) the covariates to be matched between the T2D and control groups $(\mathrm{n}=1014)$ and (4) the diabetes determinants considered in the subgroup analyses $(n=25)$. Furthermore, we have removed six remaining participants with type 1 diabetes, and one outlier with extremely high direct costs $(>€ 100000)$. Thus, the final study sample consisted of 4815 participants, among them 325 individuals with T2D and 4490 individuals without T2D.

In DEGS1, the study participants were asked by physicians in computer-assisted personal interviews about their medical history of diabetes and, in case of consent, on further details such as the current treatment or the presence of complications. ${ }^{22}$ The distinction between the diabetes types was made using an algorithm that included information on medication and age. The T2D group consisted of people with diagnosed T2D. These are people who have either indicated in DEGS1 that they have been diagnosed with diabetes or were currently taking diabetes medication or insulin. ${ }^{4}$ The control group consisted of participants without diagnosed T2D.

\section{Costs}

The annual direct (outpatient, inpatient, rehabilitation and medication), indirect (sick leave and early retirement) and total costs were estimated from a bottom-up approach using DEGS1 data on healthcare utilisation and productivity losses. The monetary valuation of healthcare utilisation based on DEGS1 data (direct costs) was carried out from a social perspective, mostly using published average unit costs, ${ }^{25}$ see online supplemental table S1 for further details on the unit costs. For medication costs, we used prices published in the drug catalogue 'Rote 
Liste'. ${ }^{26}$ In contrast to other health services, the participants in DEGS1 were not asked about the duration of their rehabilitation stay. Therefore, we assumed a mean duration of 30 days. ${ }^{27}$ Indirect costs (productivity losses) were estimated with the human capital approach. DEGS1 provided information on days of sick leave, early retirement and weekly working hours of the study participants, which we assessed in monetary terms with average wage rates. For this purpose, the average gross wage for fulltime and part-time employees was supplemented by employer's social security contributions. ${ }^{28} 29$

\section{Sociodemographic and clinical determinants}

We conducted subgroup analyses to estimate whether (1) the excess costs of individuals with T2D compared with individuals without diabetes vary by sociodemographic determinants and whether (2) the excess costs within the T2D group vary with respect to clinical determinants. As for sociodemographic determinants, we assessed age, sex and education level based on previous findings on determinants of healthcare utilisation in Germany. ${ }^{240-32}$ In the subgroup analyses, the excess costs of T2D were estimated separately for 18-64 years old individuals and individuals aged $\geq 65$ years. The level of education was measured in three groups-low, medium and high education, according to the 'Comparative Analysis of Social Mobility in Industrial Nations'. ${ }^{33}$ As for clinical determinants, we selected the following determinants based on previous COI studies ${ }^{18}{ }^{20}$ : diabetes duration, glycaemic index and diabetes complications.

\section{Statistical analysis}

We adjusted for group differences between the T2D and control groups using entropy balancing (EB) to provide a more precise measure of the excess costs caused by T2D. ${ }^{34}$ We used EB to calculate weights for the control group to match the covariates of the T2D group in mean and variance. With EB, many covariates can be balanced simultaneously, while the deviations from the origin are kept to a minimum, and no manual adjustments are required. ${ }^{34}$ In our analysis, the control group was adjusted for age, sex, education level, having a steady partnership, region (seven Nielsen areas), physician density (physicians per 100000 inhabitants) and 24 clinical covariates (see table 1 for the summary characteristics of all included covariates before and after EB). We did not include covariates that are known to be associated with diabetes (like body mass index or depression). ${ }^{35}$ For the subgroup analyses, we repeated EB for each subgroup separately to account for the differences within the subgroups. This approach ensured that the estimated excess costs within each subgroup can only be attributed to the corresponding determinant, which would not be possible with the EB weights from the main analysis. The subgroups age, sex and education were adjusted for the same covariates as in the main analysis, but without the respective sociodemographic determinant under consideration (eg, in the age group of 18-64 years, individuals with vs individuals without T2D were adjusted for all group differences except the covariate age). As for the clinical determinants, we adjusted the differences between the T2D subgroups for the covariates age, sex and education.

After EB, we estimated the mean costs for the T2D and control groups. We used generalised linear models (GLM) with a gamma distribution and a log link to test the statistical significance of the differences in mean costs. ${ }^{36}$ In the GLM, T2D (the binary predictor variable) is regressed on the respective cost category, weighted to account for other potential influencing covariates. Since a large number of covariates were accounted for in the EB model, no further adjustments have been made in the GLM. In an additional analysis, we have further added the matched EB covariates to the main model to test the influence of the sociodemographic determinants such as age, sex and education level on the direct and indirect costs. All costs were reported in Euros for the year 2011. Statistical significance was set at $\mathrm{p}<0.05$. The analyses were performed with Stata V.16 using the package 'ebal-



We tested in a first sensitivity analysis, whether the results remained robust if the outlier in the direct costs was included in the analysis. In a second sensitivity analysis, we conducted an unadjusted analysis to examine the effects of the group adjustment on costs. Then, third, we included the covariates that are known to be associated with diabetes in the EB model to test what impact these comorbidities have on costs. In detail, we additionally included body mass index, depression, hypertension, kidney failure, physical activity and smoker status as covariates in the EB model. At fourth and last sensitivity analysis, we tested whether indirect costs differed, if only the working-age population ( $<65$ years) was included in the analysis.

\section{RESULTS}

\section{Sample characteristics}

Table 1 provides information on the sample characteristics distribution before and after the EB adjustment and shows almost identical distributions after EB adjustment. With EB adjustment, individuals without diabetes were on average 61.6 years old as individuals with T2D. For both groups, the frequency of women $(47.7 \%)$ was slightly lower than that of men, and the majority of the individuals was in a steady partnership (82.5\% among individuals with T2D and $82.4 \%$ among individuals without diabetes respectively).

\section{Excess costs}

In total, costs were significantly increased by $28 \%$ in the T2D group compared with the control group (see table 2). The direct costs were statistically significantly increased by $39 \%$ for T2D. The indirect costs were $14 \%$ higher for T2D, but the difference between the groups was not statistically significant. In absolute terms, direct excess costs amounted to $€ 719$ and indirect excess costs 
Table 1 Sample characteristics before and after the group adjustment

\section{Covariates}

\section{Sociodemographic covariates}

Age (mean)

Sex (female, \%)

Education level (\%)

\begin{tabular}{llll}
\hline Low & 44.0 & 27.7 & 44.0 \\
\hline Medium & 37.9 & 51.8 & 37.8 \\
\hline High & 18.2 & 20.5 & 18.2 \\
\hline Partner (\%) & 82.5 & 82.0 & 82.4 \\
Region (Nielsen areas, \%) & & & \\
North-West & 13.2 & 12.7 & 13.2 \\
\hline North Rhine-Westphalia & 16.6 & 18.9 & 16.6 \\
\hline Central & 12.9 & 12.0 & 21.8 \\
East (North) & 21.9 & 17.4 & 16.0 \\
\hline East (South) & 16.0 & 14.1 & 11.1 \\
Bavaria & 11.1 & 12.8 & 8.3 \\
Baden-Wuerttemberg & 8.3 & 12.1 & \\
Physicians per 100000 inhabitants (\%) & & & 19.4 \\
Approximately 124 & 19.4 & 18.6 & 41.8 \\
Approximately 144 & 41.9 & 38.0 & 16.0 \\
Approximately 149 & 16.0 & 15.6 & 22.8 \\
Approximately 233 & 22.8 & 27.9 & \\
\hline Clical covarates & & &
\end{tabular}

\section{Clinical covariates}

\section{Health status, health behaviour (\%)}

Recognised disability

Contraceptive pill use

Hearing aid

Vision aid (glasses or lenses)

Comorbidities (\%)

Anxiety disorders (lifetime prevalence) 4.0

Arthrosis/degenerative joint disease (lifetime prevalence)

\begin{tabular}{llll}
\hline Bladder weakness (lifetime prevalence) & 25.9 & 13.7 & 25.8 \\
\hline Bronchial asthma (lifetime prevalence) & 8.0 & 7.6 & 8.0 \\
\hline Burnout syndrome (lifetime prevalence) & 3.1 & 4.0 & 3.1 \\
\hline Cancer (lifetime prevalence) & 8.6 & 6.3 & 8.6 \\
\hline Gastroduodenal ulcer (lifetime prevalence) & 11.7 & 6.4 & 9.2 \\
\hline Hepatitis (lifetime prevalence) & 9.2 & 5.4 & 13.2 \\
\hline Hysterectomy (lifetime prevalence) & 13.2 & 8.4 & 8.3 \\
\hline Injury/Poisoning (12 month prevalence) & 8.3 & 10.8 & 67.3 \\
\hline Joint pain (12month prevalence) & 67.4 & 54.3 & 11.1 \\
\hline Migraine (lifetime prevalence) & 11.1 & 10.6 & 4.9 \\
\hline Ovariectomy (lifetime prevalence) & 4.9 & 3.5 & 14.5 \\
\hline Prostatic hyperplasia (lifetime prevalence) & 14.5 & 7.1 & 3.4 \\
\hline Rheumatoid arthritis (lifetime prevalence) & 3.4 & 2.1 & \\
\hline
\end{tabular}

Continued diabetes mellitus $(n=325)$ Unmatched $(n=4490) \quad$ Matched $\left(n=325^{\star}\right)$

$\begin{array}{lll}61.6 & 48.3 & 61.6 \\ 47.7 & 49.9 & 47.7\end{array}$

Individuals with type 2 Individuals without diabetes mellitus 
Table 1 Continued

\begin{tabular}{|c|c|c|c|}
\hline \multirow[b]{2}{*}{ Covariates } & \multirow{2}{*}{$\begin{array}{l}\text { Individuals with type } 2 \\
\text { diabetes mellitus }(n=325)\end{array}$} & \multicolumn{2}{|c|}{ Individuals without diabetes mellitus } \\
\hline & & Unmatched $(n=4490)$ & Matched $\left(n=325^{\star}\right)$ \\
\hline $\begin{array}{l}\text { Sleep maintenance disorder (in the preceding } 4 \\
\text { weeks) }\end{array}$ & 69.9 & 63.7 & 69.8 \\
\hline Sleep onset insomnia (in the preceding 4 weeks) & 55.7 & 50.3 & 55.6 \\
\hline Thyroid disease (lifetime prevalence) & 30.2 & 23.0 & 30.1 \\
\hline
\end{tabular}

${ }^{*} n=4490$ observations in the control group were down-weighted in order to match the diabetes group.

to $€ 209$ among the total excess costs of $€ 927$. In the direct cost categories, the differences in outpatient costs (ie, physician costs) and medication costs were statistically significant. The largest relative difference between the T2D and control groups of $88 \%$ was found in medication costs.

The results of the adjusted GLM (only statistically significant results are discussed, please see online supplemental table S2 for further details) showed that T2D was associated with higher direct costs. Older age was associated with marginally higher direct costs. For T2D, this trend was reversed, although also only marginally. The influence of T2D on indirect costs was negative and not statistically significant. The indirect costs were significantly lower with increasing education levels. By contrast, for T2D, the difference between low and high education was significantly reversed and larger.
Following this, in all sociodemographic subgroups, we expected higher direct costs of individuals with T2D compared with individuals without diabetes, slightly decreasing direct excess costs with age and only small differences between the other subgroups. Based on the results, we did not expect high indirect excess costs except for individuals with high education.

\section{Diabetes determinants}

Overall, in the subgroup analyses of sociodemographic and clinical diabetes determinants, fewer results were statistically significant (see tables 3 and 4). The age group $\geq 65$ years was the only subgroup with statistically significant higher total costs for individuals with T2D compared with individuals without diabetes. However, in most of the subgroups, the costs were higher in the T2D group than in the control group (except for $>10$ years

Table 2 Estimated mean annual excess costs of individuals with T2D compared with individuals without diabetes mellitus (in Euro, 2011)

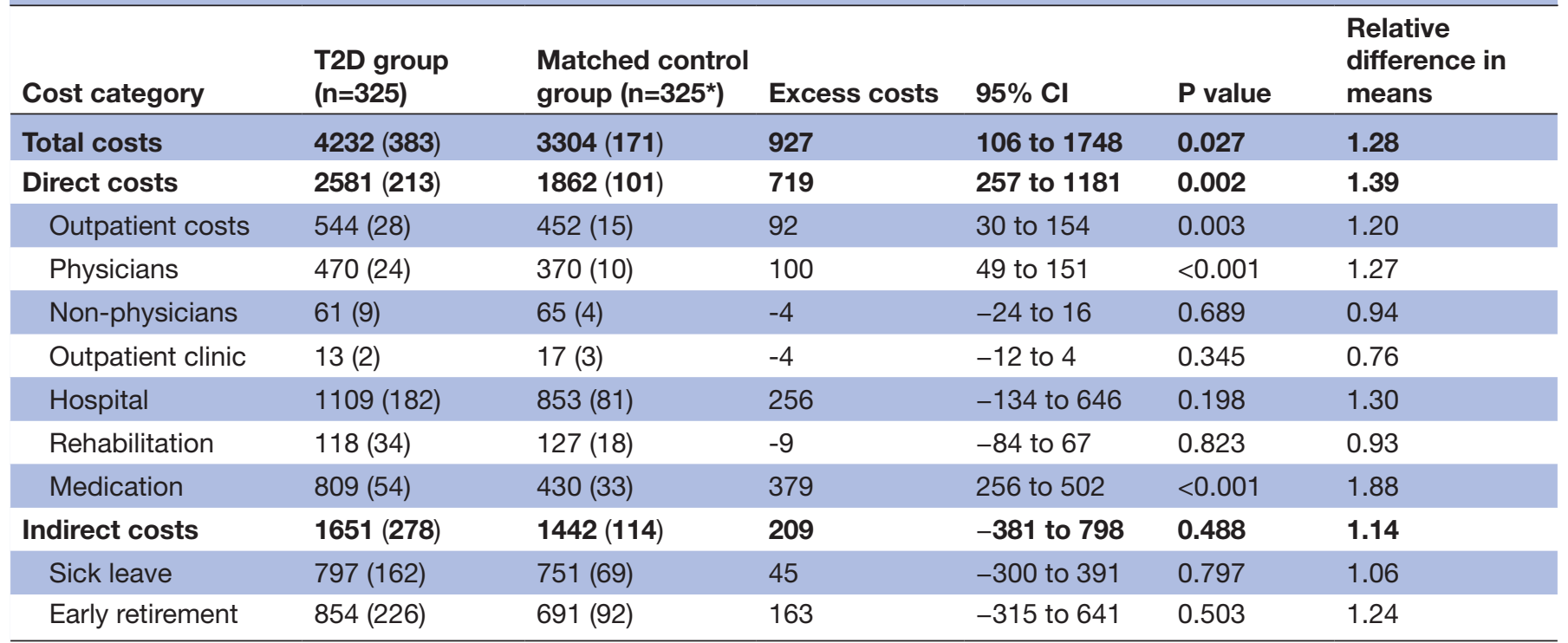

The first column shows the estimated mean costs and corresponding standard errors.

The groups were adjusted for age, sex, education level, having a steady partnership, region, physician density and 24 clinical covariates.

${ }^{*} n=4490$ observations in the control group were down-weighted in order to match the diabetes group.

T2D, type 2 diabetes. 
Table 3 Estimated mean annual excess costs of individuals with T2D compared with individuals without diabetes mellitus by sociodemographic subgroups (in Euro, 2011)

\begin{tabular}{|c|c|c|c|c|c|}
\hline Cost category & T2D group & $\begin{array}{l}\text { Matched control } \\
\text { group* }\end{array}$ & Excess costs $(95 \% \mathrm{Cl})$ & $P$ value & $\begin{array}{l}\text { Relative } \\
\text { difference in } \\
\text { means }\end{array}$ \\
\hline \multicolumn{6}{|l|}{ Sex $†$} \\
\hline \multicolumn{6}{|l|}{ Males $(n=170)$} \\
\hline Total costs & $4452(588)$ & $3543(345)$ & 910 (-427 to 2246$)$ & 0.182 & 1.26 \\
\hline Direct costs & $2622(321)$ & $2055(220)$ & $566(-197$ to 1329$)$ & 0.146 & 1.28 \\
\hline Outpatient costs & $468(34)$ & $411(17)$ & 57 (-18 to 132) & 0.134 & 1.14 \\
\hline Inpatient costs & $1186(288)$ & $1091(198)$ & 95 (-590 to 779$)$ & 0.786 & 1.09 \\
\hline Rehabilitation costs & $132(49)$ & $156(30)$ & $-24(-137$ to 88$)$ & 0.672 & 0.85 \\
\hline Medication costs & $835(72)$ & $397(52)$ & 439 (264 to 613$)$ & $<0.001$ & 2.10 \\
\hline Indirect costs & $1831(417)$ & $1487(194)$ & $343(-558$ to 1245$)$ & 0.455 & 1.23 \\
\hline Sick leave & $695(205)$ & 785 (129) & $-90(-564$ to 385$)$ & 0.711 & 0.89 \\
\hline Early retirement & $1136(353)$ & $703(148)$ & $433(-318$ to 1184$)$ & 0.259 & 1.62 \\
\hline \multicolumn{6}{|l|}{ Females $(n=155)$} \\
\hline Total costs & $3989(476)$ & 3114 (191) & $875(-130$ to 1880$)$ & 0.088 & 1.28 \\
\hline Direct costs & $2536(275)$ & $1786(119)$ & 749 (162 to 1337 ) & 0.012 & 1.42 \\
\hline Outpatient costs & $627(44)$ & $485(18)$ & 142 (49 to 236$)$ & 0.003 & 1.29 \\
\hline Inpatient costs & $1025(214)$ & $728(90)$ & 298 (-157 to 752$)$ & 0.199 & 1.41 \\
\hline Rehabilitation costs & $103(47)$ & $89(16)$ & 14 (-84 to 112$)$ & 0.776 & 1.16 \\
\hline Medication costs & $780(80)$ & $484(48)$ & 295 (111 to 479) & 0.002 & 1.61 \\
\hline Indirect costs & $1454(362)$ & $1328(137)$ & 126 (-633 to 885$)$ & 0.745 & 1.09 \\
\hline Sick leave & $908(255)$ & $693(76)$ & 215 (-307 to 737$)$ & 0.419 & 1.31 \\
\hline Early retirement & $546(269)$ & 635 (116) & $-89(-664$ to 486$)$ & 0.761 & 0.86 \\
\hline \multicolumn{6}{|l|}{ Age $\ddagger$} \\
\hline \multicolumn{6}{|l|}{$18-64$ years $(n=157)$} \\
\hline Total costs & $5684(707)$ & $4814(357)$ & 870 (-681 to 2422$)$ & 0.272 & 1.18 \\
\hline Direct costs & $2328(305)$ & $1693(147)$ & 635 (-29 to 1299) & 0.061 & 1.38 \\
\hline Outpatient costs & $537(41)$ & $446(24)$ & $91(-2$ to 184$)$ & 0.055 & 1.20 \\
\hline Inpatient costs & 1077 (269) & 795 (119) & 281 (-295 to 857$)$ & 0.339 & 1.35 \\
\hline Rehabilitation costs & $111(47)$ & $99(16)$ & $12(-87$ to 110$)$ & 0.818 & 1.12 \\
\hline Medication costs & $603(63)$ & $352(41)$ & 252 (105 to 398) & 0.001 & 1.71 \\
\hline Indirect costs & $3357(544)$ & $3122(260)$ & 235 (-946 to 1416$)$ & 0.696 & 1.08 \\
\hline Sick leave & $1588(323)$ & $1580(163)$ & $8(-700$ to 716$)$ & 0.982 & 1.01 \\
\hline Early retirement & $1768(456)$ & $1541(226)$ & 227 (-771 to 1225$)$ & 0.656 & 1.15 \\
\hline \multicolumn{6}{|l|}{$>65$ years $(n=168)$} \\
\hline Total costs & $2874(298)$ & $2138(165)$ & 736 (68 to 1404$)$ & 0.031 & 1.34 \\
\hline Direct costs & $2817(297)$ & $2122(165)$ & 695 (30 to 1360$)$ & 0.041 & 1.33 \\
\hline Outpatient costs & $550(38)$ & $475(20)$ & $76(-9$ to 160$)$ & 0.078 & 1.16 \\
\hline Inpatient costs & $1140(246)$ & 979 (127) & 161 (-382 to 705$)$ & 0.561 & 1.16 \\
\hline Rehabilitation costs & $125(49)$ & $149(31)$ & $-24(-137$ to 90$)$ & 0.682 & 0.84 \\
\hline Medication costs & $1001(83)$ & $519(61)$ & 482 (279 to 684$)$ & $<0.001$ & 1.93 \\
\hline Indirect costs & $57(28)$ & $16(6)$ & 41 (-16 to 98$)$ & 0.155 & 3.56 \\
\hline Sick leave & $57(28)$ & $16(6)$ & $41(-16$ to 98$)$ & 0.155 & 3.56 \\
\hline Early retirement & Not assessed§ & & & & \\
\hline Education levelף & & & & & \\
\hline
\end{tabular}


Table 3 Continued

\begin{tabular}{|c|c|c|c|c|c|}
\hline Cost category & T2D group & $\begin{array}{l}\text { Matched control } \\
\text { group }^{*}\end{array}$ & Excess costs $(95 \% \mathrm{Cl})$ & $P$ value & $\begin{array}{l}\text { Relative } \\
\text { difference in } \\
\text { means }\end{array}$ \\
\hline \multicolumn{6}{|l|}{ Low $(n=143)$} \\
\hline Total costs & $4132(552)$ & $3042(241)$ & 1089 (-91 to 2270$)$ & 0.071 & 1.36 \\
\hline Direct costs & 2717 (318) & $1804(149)$ & 912 (223 to 1602) & 0.009 & 1.51 \\
\hline Outpatient costs & $576(49)$ & $445(21)$ & 131 (26 to 235 ) & 0.015 & 1.29 \\
\hline Inpatient costs & $1232(261)$ & 792 (108) & 439 (-115 to 993$)$ & 0.120 & 1.56 \\
\hline Rehabilitation costs & $132(53)$ & $128(31)$ & $4(-116$ to 124$)$ & 0.948 & 1.03 \\
\hline Medication costs & $778(71)$ & $439(55)$ & 338 (163 to 514$)$ & $<0.001$ & 1.77 \\
\hline Indirect costs & $1415(414)$ & $1238(170)$ & 177 (-699 to 1053$)$ & 0.692 & 1.14 \\
\hline Sick leave & $594(215)$ & $691(111)$ & $-97(-571$ to 377$)$ & 0.689 & 0.86 \\
\hline Early retirement & $821(331)$ & 547 (129) & 274 (-423 to 971$)$ & 0.441 & 1.50 \\
\hline \multicolumn{6}{|l|}{ Medium (n=123) } \\
\hline Total costs & $4059(635)$ & $3840(370)$ & $220(-1221$ to 1661$)$ & 0.765 & 1.06 \\
\hline Direct costs & $2384(377)$ & $2060(244)$ & $324(-557$ to 1205$)$ & 0.471 & 1.16 \\
\hline Outpatient costs & 477 (36) & $416(16)$ & $60(-16$ to 137$)$ & 0.121 & 1.15 \\
\hline Inpatient costs & $1104(336)$ & $1121(227)$ & $-17(-810$ to 777$)$ & 0.967 & 0.98 \\
\hline Rehabilitation costs & $101(52)$ & $114(23)$ & $-13(-124$ to 98$)$ & 0.818 & 0.89 \\
\hline Medication costs & 703 (73) & $410(39)$ & 294 (132 to 455) & $<0.001$ & 1.71 \\
\hline Indirect costs & $1675(428)$ & $1779(203)$ & $-104(-1033$ to 824$)$ & 0.826 & 0.94 \\
\hline Sick leave & $1159(327)$ & $861(91)$ & 299 (-366 to 963$)$ & 0.378 & 1.35 \\
\hline Early retirement & $516(294)$ & 919 (187) & $-403(-1086$ to 281$)$ & 0.248 & 0.56 \\
\hline \multicolumn{6}{|l|}{ High $(n=59)$} \\
\hline Total costs & $4832(943)$ & $3101(516)$ & $1732(-375$ to 3838$)$ & 0.107 & 1.56 \\
\hline Direct costs & $2660(404)$ & $1924(286)$ & 736 (-233 to 1706$)$ & 0.137 & 1.38 \\
\hline Outpatient costs & $607(60)$ & $516(44)$ & 91 (-55 to 236$)$ & 0.222 & 1.18 \\
\hline Inpatient costs & $824(335)$ & $855(243)$ & $-31(-842$ to 780$)$ & 0.940 & 0.96 \\
\hline Rehabilitation costs & $124(86)$ & $148(46)$ & $-24(-216$ to 168$)$ & 0.807 & 0.84 \\
\hline Medication costs & 1105 (182) & $404(56)$ & 701 (328 to 1073 ) & $<0.001$ & 2.74 \\
\hline Indirect costs & $2173(737)$ & $1177(461)$ & 995 (-708 to 2699) & 0.252 & 1.85 \\
\hline Sick leave & $531(235)$ & 529 (109) & $2(-506$ to 510$)$ & 0.993 & 1.00 \\
\hline Early retirement & $1641(715)$ & $648(457)$ & 993 (-671 to 2657 ) & 0.242 & 2.53 \\
\hline
\end{tabular}

The first column shows the estimated mean costs and corresponding standard errors.

The groups were adjusted for age, sex, education level, having a steady partnership, region, physician density and 24 clinical covariates.

*The observations in the control group were down-weighted in order to match the diabetes group.

†Entropy balancing without sex.

‡Entropy balancing without age.

$\S$ No observations with costs $>0$ in the age group $65+$.

IEntropy balancing without education level.

T2D, type 2 diabetes.

diabetes duration). In the analysis of sociodemographic determinants, the total excess costs ranged from $€ 220$ for medium education to $€ 1732$ for high education. With regards to the clinical determinants, the total excess costs ranged from $€-133$ for a diabetes duration of over 10 years to $€ 1684$ for having diabetes complications. The negative total excess costs were mainly caused by negative indirect excess costs. As in the main analysis, the differences in indirect costs were not statistically significant for all subgroups. The direct costs were increased in all subgroups, but the group differences were statistically significant only for the subgroups diabetes complications, women, age $\geq 65$ years and low education. In all subgroups except for diabetes duration 4-10 versus $0-3$ years, the medication costs were increased compared with the respective control group, and except for both subgroups 
Table 4 Estimated mean annual excess costs of individuals with T2D by clinical subgroups (in Euro, 2011)

\begin{tabular}{|c|c|c|c|c|c|}
\hline Cost category & Mean costs (SE) & Mean costs (SE) & Excess costs $(95 \% \mathrm{Cl})$ & $P$ value & $\begin{array}{l}\text { Relative difference } \\
\text { in means }\end{array}$ \\
\hline \multicolumn{6}{|l|}{ Diabetes duration } \\
\hline $4-10$ vs $0-3$ years $(n=89)$ & 4-10years* & $0-3$ years & Excess costs & & \\
\hline Total costs & $4938(861)$ & $4123(692)$ & $815(-1350$ to 2980$)$ & 0.461 & 1.20 \\
\hline Direct costs & $2371(364)$ & $2169(326)$ & 203 (-754 to 1160$)$ & 0.678 & 1.09 \\
\hline Outpatient costs & $532(49)$ & $483(43)$ & $49(-80$ to 177$)$ & 0.455 & 1.10 \\
\hline Inpatient costs & $1132(314)$ & $886(264)$ & $245(-560$ to 1051$)$ & 0.550 & 1.28 \\
\hline Rehabilitation costs & $80(43)$ & $98(59)$ & $-18(-161$ to 126$)$ & 0.810 & 0.82 \\
\hline Medication costs & $628(69)$ & 702 (123) & $-74(-350$ to 202$)$ & 0.599 & 0.89 \\
\hline Indirect costs & $2567(684)$ & $1955(524)$ & $612(-1077$ to 2301$)$ & 0.477 & 1.31 \\
\hline Sick leave & $1390(496)$ & $866(247)$ & 524 (-562 to 1609) & 0.344 & 1.61 \\
\hline Early retirement & $1176(439)$ & $1088(482)$ & $88(-1190$ to 1367$)$ & 0.892 & 1.08 \\
\hline$>10$ vs $0-3$ years $(n=89)$ & $>10$ years ${ }^{\star}$ & $0-3$ years & Excess costs & & \\
\hline Total costs & $3990(603)$ & $4123(692)$ & $-133(-1933$ to 1666$)$ & 0.885 & 0.97 \\
\hline Direct costs & $2579(303)$ & $2169(326)$ & 411 (-462 to 1283$)$ & 0.356 & 1.19 \\
\hline Outpatient costs & $661(123)$ & $483(43)$ & 178 (-77 to 434$)$ & 0.171 & 1.37 \\
\hline Inpatient costs & 821 (228) & $886(264)$ & $-65(-750$ to 619$)$ & 0.851 & 0.93 \\
\hline Rehabilitation costs & $190(95)$ & $98(59)$ & 93 (-127 to 312$)$ & 0.408 & 1.94 \\
\hline Medication costs & 907 (100) & 702 (123) & $205(-105$ to 516$)$ & 0.195 & 1.29 \\
\hline Indirect costs & $1411(530)$ & $1955(524)$ & $-544(-2006$ to 918$)$ & 0.466 & 0.72 \\
\hline Sick leave & $693(217)$ & $866(247)$ & $-174(-817$ to 470$)$ & 0.597 & 0.80 \\
\hline Early retirement & $718(505)$ & $1088(482)$ & $-370(-1738$ to 998$)$ & 0.596 & 0.66 \\
\hline
\end{tabular}

Glycaemic index

\begin{tabular}{|c|c|c|c|c|c|}
\hline $\begin{array}{l}\text { HbA1c-level above vs } \\
\text { below the cut-off for } \\
\text { diabetes ( } n=63 \text { ) }\end{array}$ & $\geq 7.5 \%$ & $<7.5 \%$ * & Excess costs & & \\
\hline Total costs & $4550(809)$ & $4108(462)$ & $442(-1384$ to 2267$)$ & 0.636 & 1.11 \\
\hline Direct costs & $2799(426)$ & $2480(262)$ & $319(-662$ to 1300$)$ & 0.524 & 1.13 \\
\hline Outpatient costs & $491(52)$ & $528(33)$ & $-37(-157$ to 84$)$ & 0.550 & 0.93 \\
\hline Inpatient costs & 1177 (392) & $1096(225)$ & $81(-805$ to 966$)$ & 0.858 & 1.07 \\
\hline Rehabilitation costs & $58(58)$ & $135(41)$ & $-77(-216$ to 62$)$ & 0.278 & 0.43 \\
\hline Medication costs & $1073(114)$ & $721(53)$ & 352 (106 to 598$)$ & 0.005 & 1.49 \\
\hline Indirect costs & $1751(627)$ & $1628(333)$ & $122(-1268$ to 1513$)$ & 0.863 & 1.08 \\
\hline Sick leave & $1528(490)$ & $574(158)$ & $954(-56$ to 1964$)$ & 0.064 & 2.66 \\
\hline Early retirement & $223(222)$ & $1055(301)$ & $-832(-1564$ to -100$)$ & 0.026 & 0.21 \\
\hline \multicolumn{6}{|c|}{ Diabetes complications $\dagger$} \\
\hline $\begin{array}{l}\text { Complications vs no } \\
\text { complications } \\
(n=110)\end{array}$ & Yes & $\mathrm{No}^{*}$ & Excess costs & & \\
\hline Total costs & $5238(790)$ & $3554(460)$ & 1684 (-109 to 3476$)$ & 0.066 & 1.47 \\
\hline Direct costs & $3562(458)$ & $2157(293)$ & 1405 (339 to 2470) & 0.010 & 1.65 \\
\hline Outpatient costs & $621(51)$ & $454(31)$ & 167 (49 to 284$)$ & 0.005 & 1.37 \\
\hline Inpatient costs & $1725(402)$ & $871(251)$ & $854(-74$ to 1783$)$ & 0.071 & 1.98 \\
\hline Rehabilitation costs & $125(59)$ & $110(45)$ & $15(-130$ to 161$)$ & 0.837 & 1.14 \\
\hline Medication costs & $1090(86)$ & $722(73)$ & 368 (148 to 588 ) & 0.001 & 1.51 \\
\hline Indirect costs & $1676(518)$ & $1397(341)$ & 279 (-935 to 1493$)$ & 0.652 & 1.20 \\
\hline
\end{tabular}




\begin{tabular}{llllll}
\hline & & & & \multicolumn{2}{c}{ Relative difference } \\
Cost category & Mean costs (SE) & Mean costs (SE) & Excess costs(95\% CI) & P value & in means \\
\hline Sick leave & $779(293)$ & $464(121)$ & $315(-307$ to 937$)$ & 0.321 & 1.68 \\
Early retirement & $896(397)$ & $932(323)$ & $-36(-1038$ to 966$)$ & 0.944 & 0.96 \\
\hline
\end{tabular}

The groups were adjusted for age, sex and education level.

${ }^{*}$ The observations were down-weighted in order to match the reference group.

†Diabetes complications included diabetic nephropathy, retinopathy, neuropathy, diabetic foot, amputations, cardiovascular complications, sexual dysfunction and susceptibility to infections.

T2D, type 2 diabetes.

concerning diabetes duration, the differences were statistically significant. The highest medication excess costs were among individuals with high education. Outpatient costs were significantly increased for the respective T2D group in women, low education and individuals having diabetes complications. Furthermore, the costs of early retirement were significantly lower for individuals with a glycaemic index of haemoglobin A1c (HbA1c) $\geq 7.5 \%$ compared with individuals with a glycaemic index $<7.5 \%$.

Within the subgroups, the CIs between the respective categories (eg, men and women) always overlapped, which means that no significant group differences are to be expected. However, trends can be described, when the excess costs of the categories within each respective subgroup are compared.

\section{Sociodemographic determinants of diabetes}

In regard to total costs, the differences between men and women were only marginal. However, the indirect excess costs were higher for men than for women (23\% vs $9 \%$ ). In contrast, the direct excess costs were higher in women than in men (42\% vs $28 \%$ ). In regard to the direct cost categories, the largest difference between individuals with T2D and the control groups was found in medication costs (110\% higher in men and $61 \%$ higher in women). Although in absolute terms, the total excess costs were higher in the age group 18-64 years, the age group 65+ years incurred higher total excess costs in relative terms (34\% vs $18 \%)$. With respect to direct costs, the previously described decreasing trend in direct excess costs with older age was also found in the subgroup analysis, but only in relative terms (38\% for 18-64 years vs 33\% for $65+$ years). The absolute difference in direct costs was higher for individuals in the age group $65+$ years as a result of higher medication excess costs. The low estimates in indirect costs for the age group $65+$ years showed that this population group is usually no longer working. As for education, the total excess costs were highest with high education (56\% vs $36 \%$ for low education and $6 \%$ for medium education). Again, medication costs were among the main contributors to direct excess costs for all levels of education. For individuals with low education, inpatient and outpatient excess costs were higher than with higher education levels, resulting in higher direct excess costs. Indirect excess costs were highest for high education $(85 \%$ vs $14 \%$ for low education and $-6 \%$ for medium education), which is in line with the findings from the adjusted GLM.

\section{Clinical determinants of diabetes}

Using $\leq 3$ years duration of diabetes as reference group, a longer duration was correlated with lower indirect excess costs (the relative difference in costs amounted to $+31 \%$ for $4-10$ years and $-28 \%$ for $>10$ years). However, all direct cost categories except for inpatient costs were higher with a diabetes duration of $>10$ years compared with 4-10 years. A glycaemic index of $\geq 7.5 \%$ was related to an increase by $13 \%$ in direct costs and by $8 \%$ in indirect costs, as compared with HbA1c levels below $7.5 \%$. It was striking that with a glycaemic index of $\geq 7.5 \%$, the indirect costs due to sick leave were strongly increased by $166 \%$, while the costs of early retirement were significantly decreased by $79 \%$. Having diabetes complications was associated with $65 \%$ higher direct costs, mainly due to inpatient costs, which were $98 \%$ higher compared with individuals without diabetes complications. Indirect costs were also increased by $20 \%$ with diabetes complications, due to days of sick leave.

\section{Sensitivity analyses}

First, in the sensitivity analysis with the outlier with aboveaverage high direct costs (online supplemental table S3.1), the direct excess costs were higher than in the main analysis due to inpatient excess costs, which can be explained by the fact that the outlier was in the T2D group. The results did not change with regards to statistical significance or the direction of the effects. Second, in the unadjusted analysis (online supplemental table S3.2), the excess costs were generally higher than in the main analysis, especially for direct excess costs. Contrary to the main analysis, the inpatient and early retirement excess costs were statistically significant. Furthermore, the direction of the effect was different for the excess costs of nonphysicians, rehabilitation and sick leave. However, the correlation was not statistically significant. Third, online supplemental table S3.3 shows that the costs differ less between the T2D and the control groups, when they are additionally adjusted for the covariates that are known to be associated with diabetes. The direction of the effects did not change, but the results for total excess costs were 
no longer significant. Fourth, when only individuals in the working age were included in the analysis (online supplemental table S3.4), the indirect excess costs were marginally lower than in the main analysis ( $8 \%$ vs $14 \%$ increase in indirect costs for T2D vs control groups) but only in relative terms.

\section{DISCUSSION \\ Main findings}

As far as we know, this is the first population-based study to estimate the direct and indirect excess costs of T2D in Germany. Furthermore, this study investigated whether the excess costs of T2D differ between sociodemographic and clinical subgroups. T2D was associated with 1.28 times higher total costs. The high share of direct excess costs of approximately $78 \%$ in total excess costs, compared with indirect excess costs, is in line with earlier research. ${ }^{1011}$

\section{Direct excess costs}

In this study, the direct costs of T2D were 1.4 times higher, that is, below the range we found in previous studies from Germany (approximately 1.5 to 3.0 ) ${ }^{14-20}$ and the findings from a global systematic review (approximately 1.5 to 4.1).$^{38}$ In accordance with previous findings, medication excess costs were besides inpatient excess costs the main contributor to direct excess costs. ${ }^{1415172039}$ The inpatient excess costs also accounted for a high proportion of the direct excess costs but were not statistically significant. In general, the direct costs were highest for diabetes complications, which is also in line with previous studies. ${ }^{18} 2039$ These findings suggest that a large share of the estimated costs of T2D were mainly due to the consequences and complications of diabetes. The subgroup analysis by HbAlc groups supported the findings that a good glycaemic control is correlated with lower direct excess costs. ${ }^{20} 3940$ We found that a longer diabetes duration incurred higher direct excess costs. However, our results did not support the findings that the costs at the beginning, right after the diagnosis, are also increased. ${ }^{20}$ As for sociodemographic determinants, women had higher direct excess costs than men, which is consistent with the findings that women use healthcare services more often than men. ${ }^{24}{ }^{32}$ In line with previous findings, ${ }^{14} 17$ we observed a slightly negative trend with increasing age with respect to the relative difference in costs, although this was not confirmed in absolute terms. The impact of education on the excess costs of T2D needs further investigation. Individuals with high education had the highest medication excess costs, which could result from a better adherence to the diabetes treatment with higher education. $^{41}$

\section{Indirect excess costs}

In addition to direct excess costs, indirect excess costs were also analysed. In this study, the indirect costs of T2D were 1.1 times higher compared with controls, whereas preceding findings from Germany ranged between approximately $1.4^{17}$ and $2.1 .^{20}$ In the main analysis, as well as in the subgroup analyses, the differences in indirect costs were not statistically significant. The share of early retirement excess costs in indirect excess costs was with approximately $78 \%$ higher than that of sick leave excess costs. To our knowledge, none of the previous German COI studies investigated the impact of sociodemographic and clinical determinants on indirect excess costs. Sociodemographic determinants like education level and being male were related to higher income and productivity, which could explain the increased indirect excess costs for a higher education level and men compared with lower education levels or women. ${ }^{42}$ Furthermore, early retirement costs were significantly lower with a glycaemic index of $\mathrm{HbA1c} \geq 7.5 \%$. The reasons for this still need to be investigated, but it is most likely that no meaningful interpretable frequencies of occurrence exist, as it is generally very rare to receive early retirement benefits.

\section{Context with other studies}

In general, a reason for the lower results in our study compared with previous national and international evidence could be that we have used population-based survey data while other studies used data from patient samples. The latter are generally less healthy than population-based samples and have correspondingly higher costs. Furthermore, the costs may have been underestimated in the T2D group as severe cases are often underrepresented in population surveys. In addition, some studies also included individuals with type 1 diabetes, which could result in higher excess costs, especially for the younger age groups. Another explanation for the lower excess costs compared with previous findings might be that we have adjusted the group differences for multiple sociodemographic and clinical covariates simultaneously. To our knowledge, the preceding studies from Germany mainly conducted age-standardised and sex-standardised analyses, and only one has also adjusted for clinical covariates. ${ }^{15}$ In fact, the study with the lowest relative difference in direct costs has adjusted for the most covariates. ${ }^{15}$ The studies that used claims data from the AOK might have estimated higher excess costs than in our study because of the different insured structure (among others lower income and higher morbidity). ${ }^{43}$ Overall, the comparison with previous findings and the findings from the sensitivity analyses suggest that the comorbidities, in addition to the diabetic complications, were the main cause for high excess costs. Only the excess costs for medication and physicians remained robust.

\section{Limitations}

With DEGS1, we have used nationally representative data of high quality. We have conducted a completecase analysis, which is why the prevalence of T2D in our sample was slightly lower compared with the original DEGS1 sample. T2D was assessed by physicians in a standardised interview or on the basis of the documented use of antidiabetics. However, the results could be biased 
downwards by individuals with undiagnosed diabetes in the control group (ie, a glycaemic index above the normal threshold), who may have incurred increased costs due to untreated diabetes. Further analyses to investigate the excess costs of undiagnosed diabetes are useful and planned with the DEGS1 data, but they would go beyond the scope of this publication. The costs were estimated based on self-reported data on health service utilisation and productivity losses in the last 12 months prior to the interview, which bears the risk of reporting bias and recall bias. Although survey data have the advantage that they contain comprehensive information on healthcare utilisation and working status that can be used to estimate direct and indirect costs, some cost categories, such as dialysis treatment costs, were not assessed in this study. Furthermore, survey data contain a lot of additional information on sociodemographic and clinical characteristics of the study sample. Using these, we thoroughly adjusted for group differences between the T2D and control groups using an innovative method and investigated the correlates of sociodemographic and clinical determinants with the excess costs. Nevertheless, the estimates could be influenced by unobservable factors. With regard to the subgroup analyses, the sample sizes were partly small as a result of the complete-case analysis, resulting in wide distributions and fewer significant associations in the excess costs.

\section{CONCLUSION}

The costs of T2D were significantly increased, mainly due to direct costs. In particular, the increased medication intake in the T2D group accounted for a substantial share of the excess costs. In summary, our findings suggest that the complications and comorbid diseases of diabetes were the main contributors to the high excess costs, and not the disease itself. This might also indicate that holistic care approaches like disease management programmes might be beneficial for individuals with T2D. It is important to adjust group differences in future studies in order to precisely estimate the excess costs of T2D.

\section{Author affiliations}

${ }^{1}$ Department of Health Economics and Health Services Research, University Medical Center Hamburg-Eppendorf, Hamburg, Germany

${ }^{2}$ Department of Epidemiology and Health Monitoring, Robert Koch Institute, Berlin, Germany

Contributors $\mathrm{HK}$ designed the study and statistical analysis with substantial input from the other authors. AR, JB, CS, CB and AK contributed to the acquisition of data for the article. HK conducted the statistical analysis. HK and AK interpreted the data of the work with input from the other authors. HK has drafted the article with input from AR, JB, CS, HHK, CB and AK. All authors have critically revised the article for important intellectual content. All authors approved the final article.

Funding The DEGS1 study was carried out by the Robert Koch Institute as part of the Federal Health Monitoring in Germany on behalf of the Federal Ministry of Health. The ministry finances the Robert Koch Institute and gives substantial funds for the Federal Health Monitoring. Three of the authors are employees of the Robert Koch Institute. The funders had no role in study design, data collection and analysis, decision to publish or preparation of the manuscript. Award/grant number: NA.
Competing interests None declared.

Patient and public involvement statement In this study, we do not collect data from patients. Therefore, patients were not involved in the development of the research question, outcome measures, design, recruitment and conduct of the study. The study was accompanied by intensive public relations work. The key results were made available to the public through open access publications and health reports and aggregated data is freely accessible via the information system of the Federal Health Reporting System.

Patient consent for publication Not required.

Ethics approval The DEGS1 study protocol was consented with the Federal and State Commissioners for Data Protection and approved by the CharitéUniversitätsmedizin Berlin ethics committee in September 2008 (No. EA2/047/08). Participants provided written informed consent prior to the interview and examination.

Provenance and peer review Not commissioned; externally peer reviewed.

Data availability statement Data are available upon reasonable request. The 'Health Monitoring' Research Data Centre at the Robert Koch Institute (RKI) is accredited by the German Data Forum according to uniform and transparent standards (http://www.ratswd.de/en/data-infrastructure/rdc). The DEGS1 data set is freely accessible on application to interested scientists as de facto anonymized data for scientific secondary analysis. More detailed information on access, application forms and guidelines can be obtained from datennutzung@rki.de.

Supplemental material This content has been supplied by the author(s). It has not been vetted by BMJ Publishing Group Limited (BMJ) and may not have been peer-reviewed. Any opinions or recommendations discussed are solely those of the author(s) and are not endorsed by BMJ. BMJ disclaims all liability and responsibility arising from any reliance placed on the content. Where the content includes any translated material, BMJ does not warrant the accuracy and reliability of the translations (including but not limited to local regulations, clinical guidelines, terminology, drug names and drug dosages), and is not responsible for any error and/or omissions arising from translation and adaptation or otherwise.

Open access This is an open access article distributed in accordance with the Creative Commons Attribution Non Commercial (CC BY-NC 4.0) license, which permits others to distribute, remix, adapt, build upon this work non-commercially, and license their derivative works on different terms, provided the original work is properly cited, appropriate credit is given, any changes made indicated, and the use is non-commercial. See: http://creativecommons.org/licenses/by-nc/4.0/.

\section{ORCID iDs}

Hannah König http://orcid.org/0000-0002-4515-8405

Jens Baumert http://orcid.org/0000-0003-1399-6874

Christian Schmidt http://orcid.org/0000-0002-8419-8856

Christian Brettschneider http://orcid.org/0000-0002-5280-1075

\section{REFERENCES}

1 World Health Organization. Global report on diabetes 2016.

2 GBD 2017 Disease and Injury Incidence and Prevalence

Collaborators. Global, regional, and national incidence, prevalence, and years lived with disability for 354 diseases and injuries for 195 countries and territories, 1990-2017: a systematic analysis for the global burden of disease study 2017. Lancet 2018;392:1789-858.

3 NCD Risk Factor Collaboration (NCD-RisC). Worldwide trends in diabetes since 1980: a pooled analysis of 751 population-based studies with 4.4 million participants. Lancet 2016;387:1513-30.

4 Heidemann C, Du Y, Paprott R, et al. Temporal changes in the prevalence of diagnosed diabetes, undiagnosed diabetes and prediabetes: findings from the German health interview and examination surveys in 1997-1999 and 2008-2011. Diabet Med 2016;33:1406-14.

5 Rathmann W, Scheidt-Nave C, Roden M, et al. Type 2 diabetes: prevalence and relevance of genetic and acquired factors for its prediction. Dtsch Arztebl Int 2013;110:331.

6 Goffrier B, Schulz M, Bätzing-Feigenbaum J. Administrative Prävalenzen und Inzidenzen des diabetes mellitus von 2009 bis 2015. Zentralinstitut für die kassenärztliche Versorgung in Deutschland (Zi). Versorgungsatlas-Bericht 2017.

7 Tamayo T. Prävalenz und Inzidenz von diabetes mellitus in Deutschland 2016.

8 Schmidt C, Reitzle L, Dreß J, et al. Prävalenz und Inzidenz des dokumentierten diabetes mellitus - Referenzauswertung für 
die Diabetes-Surveillance auf basis von Daten aller gesetzlich Krankenversicherten. Bundesgesundheitsblatt Gesundheitsforschung Gesundheitsschutz 2020;63:93-102.

9 Tönnies T, Röckl S, Hoyer A, et al. Projected number of people with diagnosed type 2 diabetes in Germany in 2040. Diabet Med 2019;36:1217-25.

10 Seuring T, Archangelidi O, Suhrcke M. The economic costs of type 2 diabetes: a global systematic review. Pharmacoeconomics 2015;33:811-31.

11 Afroz A, Alramadan MJ, Hossain MN, et al. Cost-Of-Illness of type 2 diabetes mellitus in low and lower-middle income countries: a systematic review. BMC Health Serv Res 2018;18:972.

12 Nationale Diabetes-Surveillance am Robert Koch-Institut. Diabetes in Deutschland - Bericht Der Nationalen Diabetes-Surveillance 2019. Berlin: Robert Koch-Institut, 2019.

13 Statistisches Bundesamt. Krankheitskosten: diabetes mellitus, 2015, 2020. Available: https://www-genesis.destatis.de/genesis/online? operation=previous\&levelindex $=1 \&$ step $=1 \&$ titel=Ergebnis\&levelid = 1592557372049\&acceptscookies=false\#abreadcrumb [Accessed 19 Jul 2020].

14 Jacobs E, Hoyer A, Brinks R, et al. Healthcare costs of type 2 diabetes in Germany. Diabet Med 2017;34:855-61.

15 Bächle C, Claessen $\mathrm{H}$, Andrich S, et al. Direct costs in impaired glucose regulation: results from the population-based heinz Nixdorf recall study. BMJ Open Diabetes Res Care 2016;4:e000172.

16 Müller N, Heller T, Freitag MH, et al. Healthcare utilization of people with type 2 diabetes in Germany: an analysis based on health insurance data. Diabet Med 2015;32:951-7.

17 Köster I, von Ferber L, Ihle P, et al. The cost burden of diabetes mellitus: the evidence from Germany--the CoDiM study. Diabetologia 2006;49:1498-504.

18 Köster I, Hauner H, von Ferber L. [Heterogeneity of costs of diabetic patients: the Cost of Diabetes Mellitus Study]. Dtsch Med Wochenschr 2006;131:804-10.

19 Köster I, Huppertz E, Hauner H, et al. Direct costs of diabetes mellitus in Germany - CoDiM 2000-2007. Exp Clin Endocrinol Diabetes 2011;119:377-85.

20 Ulrich S, Holle R, Wacker M, et al. Cost burden of type 2 diabetes in Germany: results from the population-based KorA studies. BMJ Open 2016;6:e012527.

21 Gößwald A, Lange M, Dölle R, et al. [The first wave of the German Health Interview and Examination Survey for Adults (DEGS1): participant recruitment, fieldwork, and quality management]. Bundesgesundheitsblatt Gesundheitsforschung Gesundheitsschutz 2013;56:611-9.

22 Scheidt-Nave C, Kamtsiuris P, Gößwald A, et al. German health interview and examination survey for adults (DEGS) - design, objectives and implementation of the first data collection wave. BMC Public Health 2012;12:730.

23 Kamtsiuris $\mathrm{P}$, Lange M, Hoffmann R, et al. [The first wave of the German Health Interview and Examination Survey for Adults (DEGS1): sample design, response, weighting and representativeness]. Bundesgesundheitsblatt Gesundheitsforschung Gesundheitsschutz 2013;56:620-30.

24 Rattay P, Butschalowsky H, Rommel A, et al. [Utilization of outpatient and inpatient health services in Germany: results of the German
Health Interview and Examination Survey for Adults (DEGS1)]. Bundesgesundheitsblatt Gesundheitsforschung Gesundheitsschutz 2013;56:832-44

25 Bock JO. [Calculation of standardised unit costs from a societal perspective for health economic evaluation]. Gesundheitswesen (Bundesverband der Ärzte des Öffentlichen Gesundheitsdienstes 2015;77:53-61.

26 Liste R. Arzneimittelinformationen für Deutschland. Rote Liste Service $\mathrm{GmbH}, 2011$.

27 Deutsche Rentenversicherung Bund. Statistik Der Deutschen Rentenversicherung. rehabilitation 2011. Würzburg, 2012

28 Statistisches Bundesamt, Verdienste und Arbeitskosten. Arbeitskosten im Produzierenden Gewerbe und im Dienstleistungsbereich- Ergebnisse für Deutschland - 2008. Wiesbaden, 2014.

29 Statistisches Bundesamt, Verdienste und Arbeitskosten. Arbeitnehmerverdienste. 4. Vierteljahr 2011. Wiesbaden, 2012.

30 Hoebel J, Rattay P, Prütz F, et al. Socioeconomic status and use of outpatient medical care: the case of Germany. PLoS One 2016;11:e0155982.

31 Luppa M. Frequent attenders in the German healthcare system: determinants of high utilization of primary care services. results from the cross-sectional German health interview and examination survey for adults (degS). BMC Fam Pract 2019.

32 Rommel A, Kroll LE. Individual and regional determinants for physica therapy utilization in Germany: multilevel analysis of national survey data. Phys Ther 2017;97:512-23.

33 Brauns H, Scherer S, Steinmann S. The CASMIN educational classification in international comparative research, in advances in cross-national comparison. Springer, 2003: 221-44.

34 Hainmueller J. Entropy balancing for causal effects: a multivariate reweighting method to produce balanced samples in observational studies. Political Analysis 2012;20:25-46.

35 Gabrys L. Diabetes-Surveillance in Deutschland-Auswahl und definition von Indikatoren 2018.

36 Glick HA. Economic evaluation in clinical trials. Oxford: OUP, 2010.

37 Hainmueller J, Xu Y. ebalance: A Stata Package for Entropy Balancing. J Stat Softw 2013;54:12

$38 \mathrm{Ng}$ CS, Lee JYC, Toh MP, et al. Cost-Of-Illness studies of diabetes mellitus: a systematic review. Diabetes Res Clin Pract 2014;105:151-63.

39 Mata-Cases M, Casajuana M, Franch-Nadal J, et al. Direct medica costs attributable to type 2 diabetes mellitus: a population-based study in Catalonia, Spain. Eur J Health Econ 2016;17:1001-10.

40 Aagren M, Luo W. Association between glycemic control and shortterm healthcare costs among commercially insured diabetes patients in the United States. J Med Econ 2011;14:108-14.

41 Kirkman MS, Rowan-Martin MT, Levin R, et al. Determinants of adherence to diabetes medications: findings from a large pharmacy claims database. Diabetes Care 2015;38:dc142098-9.

42 Statistisches Bundesamt. Arbeitsmarkt auf einen Blick- Deutschland und Europa 2018.

43 Hoffmann F, Icks A. [Structural differences between health insurance funds and their impact on health services research: results from the Bertelsmann Health-Care Monitor]. Gesundheitswesen 2012;74:291-7. 